\title{
AN IMPROVED ESTIMATE FOR THE BLOCH NORM OF FUNCTIONS IN DOOB'S CLASS
}

\author{
J. S. HWANG AND D. C. RUNG
}

ABSTRACr. For any fixed $0<\rho<2 \pi, \mathscr{D}(\rho)$ is the family of all holomorphic functions in $\Delta$ which satisfy (i) $f(0)=0$, and (ii) $\lim _{z \rightarrow r}|f(z)|>1$, for all $\tau$ lying on some arc $\Gamma_{f} \subseteq \partial \Delta$ with arclength $\left|\Gamma_{f}\right|>\rho$. We showed that for each $f \in \mathscr{D}(\rho)$ there exists a point $z_{f} \in \Delta$ at which

$$
\left|f^{\prime}\left(z_{f}\right)\right|\left(1-\left|z_{f}\right|^{2}\right)>\frac{2}{e} \frac{\sin (\pi-(\rho / 2))}{(\pi-(\rho / 2))} .
$$

In this paper we improve this estimate by replacing the quantity $\pi-(\rho / 2)$ with a value $\theta(\rho)$ which lies between 0 and $\pi-(\rho / 2)$ and so improves the estimate. The value $\theta(\rho)$ is defined as the (unique) solution in this interval of the equation $F_{\rho}(\theta)=\log (\cot (\rho / 4) \cot (\theta / 2))-\theta / \sin \theta=0$.

1. In a series of papers ([4]-[8]) J. L. Doob introduced a family $\mathscr{D}(\rho)$ of holomorphic functions in the unit disc $\Delta$, whose boundary we denote by $\partial \Delta$. For any fixed $0<\rho<2 \pi, \mathscr{D}(\rho)$ is the family of all holomorphic functions in $\Delta$ which satisfy (i) $f(0)=0$, and (ii) $\underline{\lim }_{z \rightarrow \tau}|f(z)|>1$, for all $\tau$ lying on some arc $\Gamma_{f} \subseteq \partial \Delta$ with arclength $\left|\Gamma_{f}\right| \geqslant \rho$. Doob posed the question as to whether the set of Bloch norms $\left\{\|f\|_{\mathscr{B}}=\sup _{z \in \Delta}\left|f^{\prime}(z)\right|\left(1-|z|^{2}\right)\right\}_{f \in \Phi(\rho)}$ has a positive lower bound. We showed in [10] that for each $f \in \mathscr{D}(\rho)$ there exists a point $z_{f} \in \Delta$ at which

$$
\left|f^{\prime}\left(z_{f}\right)\right|\left(1-\left|z_{f}\right|^{2}\right) \geqslant \frac{2}{e} \frac{\sin (\pi-(\rho / 2))}{(\pi-(\rho / 2))} .
$$

In this paper we improve this estimate by replacing the quantity $\pi-(\rho / 2)$ with a value $\theta(\rho)$ which lies between 0 and $\pi-(\rho / 2)$. The value $\theta(\rho)$ is defined as the (unique) solution in this interval of the equation

$$
F_{\rho}(\theta)=\log (\cot (\rho / 4) \cot (\theta / 2))-\frac{\theta}{\sin \theta}=0 .
$$

Functions in $\mathscr{D}(\rho)$ produce upper estimates to the various Bloch constants [2]. For $f$ holomorphic in $\Delta$ set $b(f)=\sup \left\{r \mid\right.$ there exists a domain $\Delta_{1} \subseteq \Delta$ such that $f$ is univalent on $\Delta$ and $f\left(\Delta_{1}\right)$ contains a disc of radius $r$. If $\mathscr{B}$ denotes the family of holomorphic functions in $\Delta$ normalized by $\left|f^{\prime}(0)\right| \geqslant 1$, then the Bloch constant $B$ is defined as

$$
B=\inf b(f), \quad f \in \mathscr{B}
$$

Received by the editors September 19, 1979.

AMS (MOS) subject classifications (1970). Primary 30A42; Secondary 30A72.

Key words and phrases. Bloch, Landau constant, differential maximal principle. 
If $\mathscr{B}_{s}$ denotes the subfamily of $\mathscr{B}$ of all univalent functions then $B_{s}=\inf b(f)$, $f \in \mathscr{B}_{s}$. It is known that $\sqrt{3} / 4<B<.472 ; .544<B_{s}<.658$. These lower estimates are due to Heins [9] and Landau [11], respectively, while the upper estimates are due to Ahlfors and Grunsky [1] and R. Robinson [13], respectively. With no loss of generality the normalization $\left|f^{\prime}(0)\right|>1$ can be relaxed. If $L$ is a Möbius transformation of $\Delta$ onto $\Delta$ taking 0 into $z$ then we have both $b(f \circ L)=b(f)$ and $(f \circ L)^{\prime}(0)=\left(1-|z|^{2}\right)\left|f^{\prime}(z)\right|$. So we enlarge $\mathscr{B}$ (and $\left.\mathscr{B}_{s}\right)$ by replacing $\left|f^{\prime}(0)\right|>1$ by $\left|f^{\prime}\left(z_{f}\right)\right|\left(1-\left|z_{f}\right|^{2}\right) \geqslant 1$, for some $z_{f} \in \Delta$. The constants $B$ and $B_{s}$ remain unchanged. From our previous results as stated in (1.0) we see that $f \in \mathscr{D}(\rho)$ implies

$$
\left(\frac{e}{2} \frac{(\pi-(\rho / 2))}{\sin (\pi-(\rho / 2))}\right) f \in \mathscr{B},
$$

and so upper estimates for $B$ (and $B_{s}$ ) can be obtained from functions in $\mathscr{D}(\rho)$. Any improvement in the constant $(e / 2)(\pi-(\rho / 2)) / \sin (\pi-(\rho / 2))$ should be of some interest.

2. Main result. If $A \subseteq \Delta$, let $\partial A$ denote the topological boundary of $A$. If $\partial A \cap \partial \Delta$ contains an arc $\Gamma$ then, as usual, let $\omega(z, \Gamma, A)$ denote the harmonic measure at $z$ of $\Gamma$ relative to $A$. If $A=\Delta$ then we define the lens-shaped domain in $\Delta$ by

$$
S(\alpha, \Gamma)=\{z \in \Delta \mid \omega(z, \Gamma, \Delta)>(\pi-\alpha) / \pi\}, \quad 0<\alpha<\pi
$$

It is easy to show that $\partial S(\alpha, \Gamma) \cap \Delta$ makes the angle $\alpha$ with $\partial \Delta$. If $\alpha=\pi-(\rho / 2)$ then $\partial S(\alpha, \Gamma)$ contains the origin. The proof of the main result is based upon a sharpened form of the Lehto-Virtanen differential two constant theorem [12] due to S. Dragosh and D. C. Rung [3]. For completeness we state a less general version suitable for our needs. It is similar to the version used in [10].

TheOREM D-R. Let $f$ be meromorphic in $\Delta$. Fix a domain of the form $S(\alpha, \Gamma)$ and suppose

(i) $\sup _{z \in S(\alpha, \Gamma)}|f(z)|=M<\infty$;

(ii) there exists a point $q \in \partial S(\alpha, \Gamma) \cap \Delta$ at which $|f(q)|=M$;

(iii) for each $\tau \in \Gamma, \overline{\lim }_{z \rightarrow \tau}|f(z)|<m<M$.

Then

$$
\left|f^{\prime}(q)\right|\left(1-|q|^{2}\right) \geqslant\left(\frac{2 \sin \alpha}{\alpha}\right) M \log \frac{M}{m}
$$

In preparation for the main result we need a few more details. For any fixed $0<\rho<2 \pi$, denote the (unique) root of $\log [\cot (\rho / 4) \cot (\theta / 2)]-\theta / \sin \theta=0$ lying in $(0, \pi-(\rho / 2))$ by $\theta(\rho)$. On this interval the first term of the equation decreases from $+\infty$ to 0 while the left side increases from 1 to $(\pi-\rho / 2) / \sin (\pi-(\rho / 2))$. Given an arc $\Gamma \subseteq \partial \Delta$, with midpoint $\tau$, let $r_{\tau}$ denote the radius to $\tau$ and set $\hat{S}(\alpha, \Gamma)=S(\alpha, \Gamma) \cup r_{\tau}, 0<\alpha<\pi$. 
TheOREM 1. Suppose $f \in \mathscr{D}(\rho)$ for some fixed $\rho, 0<\rho<2 \pi$, and some arc $\Gamma_{f} \subseteq \partial \Delta$. Then there exists at least one point $z_{f} \in \hat{S}\left(\theta(\rho), \Gamma_{f}\right)$ at which

$$
\left|f^{\prime}\left(z_{f}\right)\right|\left(1-\left|z_{f}\right|^{2}\right)>\frac{2}{e} \frac{\sin \theta(\rho)}{\theta(\rho)} \text {. }
$$

Proof. We may suppose $\Gamma_{f}$ is symmetric about $z=1$ so that $r_{\tau}$ is the segment $0<x<1$. To the contrary suppose for all $z \in \hat{S}\left(\theta(\rho), \Gamma_{f}\right)$

$$
\left|f^{\prime}(z)\right|\left(1-|z|^{2}\right)<\frac{2}{e} \frac{\sin \theta(\rho)}{\theta(\rho)}
$$

Let $x^{*}$ be the intersection of $\partial S\left(\theta(\rho), \Gamma_{f}\right)$ and $[0,1)$. We are going to show first that (2.2) and Theorem D-R imply $\left|f\left(x^{*}\right)\right|>1 / e$; and secondly a straightforward estimate from (2.2) implies $\left|f\left(x^{*}\right)\right|<1 / e$. To show the first inequality we put $g=1 / f$ and let $\Gamma_{\theta}$ denote the subarc of $\Gamma_{f}$ with endpoints $e^{-i \theta}$ and $e^{i \theta}, 0<\theta<$ $\rho / 2$. Put $M(\theta)=\sup |g(z)|, z \in S\left(\theta(\rho), \Gamma_{\theta}\right)$. Choose any value $\theta$ for which $M(\theta)$ is finite. Apply Theorem D-R to $g$ on the domain $S\left(\theta(\rho), \Gamma_{\theta}\right)$. In this situation $m=1$ and $M=M(\theta)$ and so we conclude that for some $q_{\theta} \in \partial S\left(\theta(\rho), \Gamma_{\theta}\right) \cap \Delta$

$$
\left|g^{\prime}\left(q_{\theta}\right)\right|\left(1-\left|q_{\theta}\right|^{2}\right)>\frac{2 \sin (\theta(\rho))}{\theta(\rho)} M(\theta) \log M(\theta) .
$$

If we remember $g=1 / f$ then (2.3) together with assumption (2.2) gives

$$
\frac{2 \sin \theta(\rho)}{\theta(\rho)} \frac{\log M(\theta)}{M(\theta)}<\left|f^{\prime}\left(q_{\theta}\right)\right|\left(1-\left|q_{\theta}\right|^{2}\right)<\frac{2}{e} \frac{\sin \theta(\rho)}{\theta(\rho)},
$$

or

$$
\frac{\log M(\theta)}{M(\theta)}<\frac{1}{e}
$$

In the interval $[1, \infty)$ the function $\log x / x$ has a single maximum value of $1 / e$ at $x=e$. The finite (and infinite) values of $M(\theta)$ form a connected set, which because of (2.4) lies either in $[1, e)$ or $(e, \infty]$. But $f \in \mathscr{D}(\rho)$ implies that $M(\theta)$ is close to 1 for small values of $\theta$ and so we conclude that $M(\theta)<e$ for all values of $0<\theta<\rho / 2$. In particular $1 /\left|g\left(x^{*}\right)\right|=\left|f\left(x^{*}\right)\right|>1 / e$. In the other direction we estimate $f\left(x^{*}\right)$ by integrating $f^{\prime}(x)$ along the interval $\left[0, x^{*}\right]$. Under assumption (2.2) and remembering that $f(0)=0$ we have

$$
\begin{aligned}
\left|f\left(x^{*}\right)\right| & <\int_{0}^{x^{*}}\left|f^{\prime}(x)\right| d x<\frac{2}{e} \frac{\sin \theta(\rho)}{\theta(\rho)} \int_{0}^{x^{*}} \frac{d x}{1-x^{2}} \\
& =\frac{1}{e} \frac{\sin \theta(\rho)}{\theta(\rho)} \log \left(\frac{1+x^{*}}{1-x^{*}}\right) .
\end{aligned}
$$

To solve for $x^{*}$ in terms of $\theta(\rho)$ and $\rho$ recall that $\partial S\left(\theta(\rho), \Gamma_{f}\right) \cap \Delta$ is part of a circle making an angle $\theta(\rho)$ with $\partial \Delta$ at $e^{-i \rho / 2}$ and $e^{i \rho / 2}$. Using the law of sines we calculate $x^{*}$ as the difference between the distance of the center of the circle determined by $\partial S\left(\theta(\rho), \Gamma_{f}\right)$ from the origin, and the radius of this circle. We obtain 
that

$$
x^{*}=\frac{\sin (\theta(\rho))-\sin (\rho / 2)}{\sin (\theta(\rho)-(\rho / 2))} .
$$

A routine use of trigonometric identities shows that

$$
\frac{1+x^{*}}{1-x^{*}}=\cot \left(\frac{\theta(\rho)}{2}\right) \cot (\rho / 4) \text {. }
$$

Because $\theta(\rho)$ was chosen so that

$$
\frac{\sin \theta(\rho)}{\theta(\rho)} \log \left[\cot \left(\frac{\theta(\rho)}{2}\right) \cot (\rho / 4)\right]=1
$$

(2.5) and (2.6) show that $f\left(x^{*}\right)<1 / e$. Thus a contradiction is reached and (2.1) is established. Because $0<\theta(\rho)<\pi-(\rho / 2)$ the monotonicity of $\sin x / x$ shows that (2.1) is a better estimate than (1.0). In fact it is a much stronger result especially for small values of $\rho$. For example when $\rho=\pi$ the constant in $(1.0)$ is $4 / e \pi \sim .468$, while in (2.1) it is $\sim .684$; if $\rho=\pi / 10$ then (1.0) gives a constant of $\sim .08$ while (2.1) gives $\sim$.345. As $\rho \rightarrow 2 \pi, \theta(\rho) \rightarrow 0$ and $(2 / e) \sin \theta(\rho) / \theta(\rho) \rightarrow 2 / e$. In this asymptotic case the constant $2 / e$ is best possible as was pointed out in [10]. Whether $(2 / e) \sin \theta(\rho) / \theta(\rho)$ is best possible in general we do not know. In Table 1 we give various values of $\rho, \theta(\rho)$ and $\kappa(\rho)=(2 / e) \sin \theta(\rho) / \theta(\rho)$. It is easy to generate extensive values of $\kappa(\rho)$ with any computer. Numbers in the table have been rounded to three places.

\begin{tabular}{c|c|c}
\multicolumn{3}{|c}{ TABLE 1 } \\
$\rho$ & $\theta(\rho)$ & $\kappa(\rho)$ \\
\hline$\pi$ & .657 & .684 \\
$4 \pi / 5$ & .839 & .653 \\
$3 \pi / 5$ & 1.06 & .606 \\
$2 \pi / 5$ & 1.33 & .538 \\
$\pi / 5$ & 1.70 & .429 \\
$\pi / 50$ & 2.35 & .224
\end{tabular}

The convergence of $\kappa(\rho)$ to 0 with $\rho$ is rather slow. We have not been able to use functions belonging to the family $\mathscr{D}(\rho)$ to obtain any improvement in the upper estimates for $B$ and $B_{S}$.

In Theorem 1 the normalization $f(0)=0$ can be replaced by $f(a)=0$ to produce a slightly more general theorem.

THEOREM 2. Let $f$ be holomorphic in $\Delta$ and suppose $f(a)=0, a \in \Delta$. Suppose further for some arc $\Gamma_{f} \subseteq \partial \Delta$ we have, for all $\tau \in \Gamma_{f}, \lim _{z \rightarrow \tau}|f(z)|>1$. Then there exists a point $z_{f} \in S\left(\pi\left(1-\omega\left(a, \Gamma_{f}, \Delta\right)\right), \Gamma_{f}\right)$ at which

$$
\left|f^{\prime}\left(z_{f}\right)\right|\left(1-\left|z_{f}\right|^{2}\right)>\kappa\left(2 \pi \omega\left(a, \Gamma_{f}, \Delta\right)\right) .
$$

Proof. It is easy to see that $g(\zeta)=f((\zeta+a) /(1+\bar{a} \zeta))$ is in $\mathscr{D}\left(2 \pi \omega\left(a, \Gamma_{f}, \Delta\right)\right)$ and of course $\left|g^{\prime}(\zeta)\right|\left(1-|\zeta|^{2}\right)=\left|f^{\prime}(z)\right|\left(1-|z|^{2}\right), z=(\zeta+a) /(1+\bar{a} \zeta)$. An application of Theorem 1 to $g(\zeta)$ proves Theorem 2. (Actually $z_{f}$ lies in a smaller 
domain but for simplicity we use the more familiar albeit larger domain $S\left(\pi\left(1-\omega\left(a, \Gamma_{f}, \Delta\right)\right), \Gamma_{f}\right)$.)

We close with several questions on the classes $\mathscr{D}(\rho)$. Can one say anything about the boundary behavior of $f$ away from the arc $\Gamma_{f}$ on which $|f|>1$ ? By applying Fatou's Theorem to $1 / f$ in a neighborhood of $T_{f}$ we see that $f$ has angular limits almost everywhere on $\Gamma_{f}$. Can the condition $\lim _{z \rightarrow \Gamma_{f}}|f(z)| \geqslant 1$ be relaxed to allow this lower limit only for certain approaches to $\Gamma_{f}$ ? And lastly, is it possible to allow $\Gamma$ to be the union of finitely many arcs-perhaps symmetrically arranged on $\partial \Delta$-and to produce a lower estimate for $\|f\|_{\mathscr{B}}$ which is better than the one obtained by considering the largest subarc of $\Gamma$ ?

\section{REFERENCES}

1. L. V. Ahlfors and H. Grunsky, Über die Blochsche Konstante, Math. Z. 42 (1937), 671-673.

2. A. Bloch, Les théorèmes de M. Valiron sur les fonctions entières, et la théorie de l'uniformisation, Ann. Fac. Sci. Univ. Toulouse 3 (1925), 1-22.

3. S. Dragosh and D. C. Rung, Normal functions bounded on arcs and a proof of the Grass cluster-value theorem, Hiroshima Math. J. 9 (1979), 303-312.

4. J. L. Doob, The boundary values of analytic functions, Trans. Amer. Math. Soc. 34 (1932), 153-170.

5. The boundary behavior of analytic functions. II, Trans. Amer. Math. Soc. 35 (1933), $718-751$.

6.

7. __ Cluster values of sequences of analytic functions, Sankhya Ser. A 25 (1963), 137-148.

8. One sided chuster value theorems, Proc. London Math. Soc. 3 (1963), 461-470.

9. M. Heins, A class of conformal metrics, Bull. Amer. Math. Soc. 67 (1961), 475-478.

10. J. S. Hwang and D. C. Rung, Proof of a conjecture of Doob, Proc. Amer. Math. Soc. 75 (1979), 231-234.

11. E. Landau, Über die Blochsche Konstante und zwei verwandte Weltkonstanten, Math. Z. 30 (1929), 608-634.

12. O. Lehto and K. I. Virtanen, Boundary behavior and normal meromorphic functions, Acta Math. 97 (1957), 47-65.

13. R. M. Robinson, The Bloch constant $\mathfrak{A}$ for a schlicht function, Duke Math. J. 1 (1935), 535-540.

acadamia Sinica, Taipei, Taiwan, Republic of China

Department of Mathematics, Pennsylvania State University, University Park, Pennsylvania 16802 